\title{
Review of Artificial Intelligence Techniques Application to Dissolved Gas Analysis on Power Transformer
}

\author{
Naveen Kumar Sharma, Member, IACSIT, Prashant Kumar Tiwari, Member, IACSIT, \\ and Yog Raj Sood, Member, IACSIT
}

\begin{abstract}
Transformers are a critical part of an electrical utility's asset base. On-line monitoring and diagnostics is a useful tool to help operators to manage their assets and make decisions on continuing operation, maintenance or replacement. Dissolved Gas Analysis (DGA) is the heart of on-line monitoring as it is a well-established method of transformer diagnosis. DGA techniques are simple, inexpensive, and widely used to interpret gases dissolved due to the deterioration of the insulating oil of power transformers and hence to diagnosis, possibility of various type of faults in power transformer. Various diagnostic criteria based on gas analysis have been developed. In this paper, the application of many AI techniques have been presented such as Artificial Neural Network (AAN), Fuzzy Interface System (FIS), Genetic Algorithm (GA), Extended Relation Function (ERF), Bayesian Network (BN), Self Organizing Map (SOM) and Discrete Wavelet Network (WNs) Transforms, which can be used to increase the efficient and accurate diagnosis for off line and on line monitoring of power transformers.
\end{abstract}

Index Terms-Power Transformer, Dissolve Gas Analysis, Artificial Intelligence Techniques.

\section{INTRODUCTION}

Power transformers play an important role in both the transmission and distribution of electrical power system. It is very essential oil-insulated components in power systems and its operational state determines the safety of the whole power system. Failure of a transformer may cause long interruptions in power supply and require expensive repairs. An incipient fault in a transformer should be detected as early as possible, preventing the transformer from further deterioration. Diagnostic approaches can be divided into two groups: on-line and off-line. Transformer ageing process of insulating oil and cellulose materials has been monitored by many different techniques such as Dissipation factor, capacitance, Breakdown voltage of oil and paper, Degree of polymerization (DP), Total combustible Gases (TCG), furan analysis, Interfacial Tension (IFT) analysis etc. Dissolved Gas Analysis is most widely method to detect incipient faults in oil filled transformer and Electrical equipment. Dissolved

Manuscript received January 29, 2011; revised July 26, 2011.

Naveen Kumar Sharma is with Electrical Engineering Department, National Institute of Technology, Hamirpur (H.P.), INDIA. (e-mail: naveen31.sharma@gmail.com).

Prashant Kumar Tiwari is with Electrical Engineering Department, National Institute of Technology, Hamirpur (H.P.), INDIA. (e-mail: prashant081.in@gmail.com).

Yog Raj Sood, Professor is with Electrical Engineering Department \& Dean (Research \& Development), National Institute of Technology, Hamirpur (H.P.), INDIA. (e-mail: yrsood@gmail.com). gas analysis is made on the basis of the standard IEC60599 standards [1]. Several diagnosis methods, such as the Rogers and Duval Triangle methods, are available to identify the different types of fault occurring in service such as arcing, partial discharges, or hot spots. DGA includes detection, quantification and characterization of the Gases. These gases, called characteristic gases, include, Hydrogen (H2), Methane (CH4), Ethane (C2H6), Ethylene (C2H4) and Acetylene $(\mathrm{C} 2 \mathrm{H} 2)$, and Carbon Oxides such as $\mathrm{CO}$ and $\mathrm{CO} 2$. The nature and the amount of the individual component gases extracted from the oil may be indicative of the type and degree of abnormality. The DGA data provides information about the condition of the transformer and advance warning of developing faults, monitoring the rate of fault development, confirm the presence of faults, conveniently scheduling repairs and monitoring of condition during overload.

Dissolved Gas Analysis (DGA) is a usual method to diagnose the fault of power transformer. There are many conventional methods used to detect transformer fault, mainly including Key Gas method Rogers Ratio Method, Dorenburgs Ratio Method, IEC method, Duval's Triangle method, Denkyoken method, CIGRE's method, Nomograph method, NBR7274 method and IS 10593:2006 method [2-9].

In classical method the interpretation of the result to diagnosis the fault in power transformer depends upon the experience of human experts. Some time a high degree of inconsistency and ambiguity have absorbed regarding interpretation of result by different human experts so there is a urgent need of development AI techniques used for DGA are explained in this paper

In this paper, AI techniques like Artificial Neural Network (AAN), Fuzzy Interface System (FIS), Genetic Algorithm (GA), Extended Relation Function (ERF), Bayesian Network (BN), Self Organizing Map (SOM) and Discrete Wavelet Network (WNs) Transforms applied to Dissolved Gas Analysis method have been discussed.

\section{Artificial NeUral Network ApPliCAtion to DGA}

The Artificial Neural Network method can be used more accurately and effective for this purpose since the hidden relationships between the fault types and dissolved gases can be recognized by ANN through training process. An ANN used with Adaptive Back-propagation learning algorithm for the fault diagnosis of power transformer.

ANN can learn and adapt to statistical distributions, extract essential characteristics from input data, and require no physical models. Since fault diagnosis is basically a process of associating an input data pattern to one or more fault conditions, ANN is an ideal tool for such a task [10]. 
In ANN apply mainly work, Input feature-selection constitutes an essential as a first step and next step of the ANN application design is to choose network topology. That should be chosen very carefully so that the input features will correctly reflects the characteristics of the problem. That should be done experimentally through a repeated process to optimize the number of hidden layers and nodes according to training performance and prediction accuracy. In transformer insulating oil dissolved key gases like H2, CH4, C2H6, $\mathrm{C} 2 \mathrm{H} 4$, and $\mathrm{C} 2 \mathrm{H} 2$ are chosen as input features in ANN. Since overheating, partial discharge and arcing are the three major fault types in power transformers; hence there will be four output patterns to be identified including the normal condition [11].

The ANNs are tools particularly adapted to help the specialists in maintenance in the activities of classification, diagnosis and decision makings, prediction etc. An important advantage of ANN-based fault diagnostics is that it can learn directly from the training samples, and update its knowledge when necessary. The highly non-linear mapping capability of neurons provides a comparable and often superior performance over fuzzy system solutions. ANN computational complexity is not too high, especially in testing (diagnosis) process. For these reasons, many studies are undertaken in the field of maintenance to evaluate the contributions of neural networks before their operational implementation. The application of ANN makes possible to reduce considerably the laboratory experiment time while networks learn how to predict properties of insulation for duration longer than those of the tests thus constituting a tool making more economic the tests of high voltage in general. ANN method is more accurately applied to Dissolved Gas Analysis since the hidden relationships between fault types and dissolved gases can be recognized by ANN through training process [12-15].

A multi-layer feed-forward artificial neural network has been proposed to identify the fault and a generalized delta rule has been used for training of network. Implementation involves the generating of training data, and design of ANN structure, training of network and testing. In this application, gas ratios become the inputs for neural network and types of faults become the outputs of the network. To start with, a three layer network has been considered, in which size of input layer is equal to number of gas ratios and size of output layer is equal to the number of different faults and the nodes in the hidden layer are selected based on experience and a schematic diagram of a three layer network is shown in Figure 1. [16].

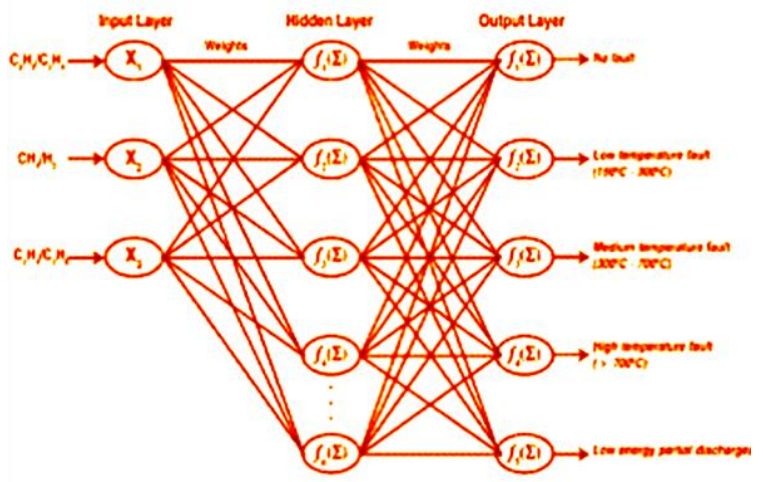

Fig.1. Multi-layer feed-forward Neural Network [16].

\section{FUZZY LOGIC APPLICATION TO DGA}

A Periodic maintenance of large power transformers fault diagnosis system has been proposed and implemented of fuzzy diagnosis to improve the conventional DGA methods. DGA technology is approved as applicable to discover internal latent transformer failure and its development trend. Therefore, whether in the domestic or international arena, DGA technology has secured a significant position in the rank of preventive testing of electrical equipments. The fuzzy logic analysis involves three successive processes, namely: Fuzzification, Fuzzy Inference and Defuzzification [17].

In the process of designing a transformer fault diagnostic system, the uncertainly shows the following two characteristics :(i) A great number of test data (including the preventive test and other tests), some expert experience and some criteria which can be directly numerated should be translated into fuzzy numbers. This process is called numerical uncertainty translation. (ii) Some expert experience and some criteria which are expressed in linguistic language cannot be numerated directly. They also need to be changed into fuzzy numbers. The process is called linguistic uncertainty translation.

In fuzzy diagnosis system we take first, the associated membership functions of fuzzy subsets were determined empirically or basically in a trial-and-error manner, while the conventional DGA diagnosis criteria were to be implicitly obeyed. And second step, a great number of previous diagnosis records of dissolved gas were mainly employed as a test purpose rather than as a development basis of the fuzzy diagnosis system. The information inherently contained in the numerical data was not fully utilized in establishing the diagnosis system. And the last step, due to the wide variety of conditions which affect the results of DGA, the diagnosis system should be continuously maintained or modified according to the cases newly obtained. However, manual knowledge acquisition and knowledge base revision out of the large numerical data are quite tedious, often resulting in a lengthy process of generating the fuzzy if-then rules and adjusting the membership functions of the corresponding fuzzy subsets [18].

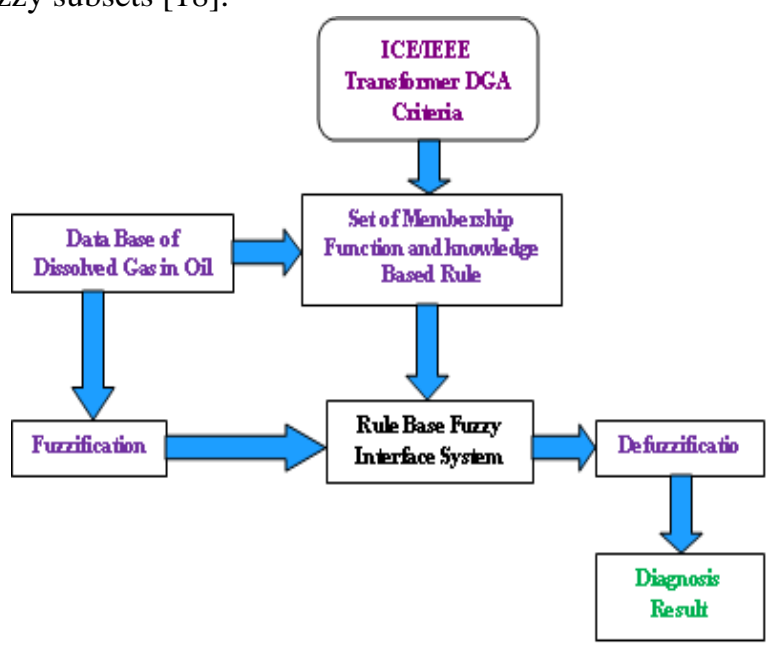

Fig.2. Structure of the Fuzzy Diagnosis System [17]

The approach is based on the interpretation of DGA data using Fuzzy Logic (FL). The proposed diagnostic method adopts indicators related to the ratios $\mathrm{C} 2 \mathrm{H} 4 / \mathrm{C} 2 \mathrm{H} 6$, 
$\mathrm{C} 2 \mathrm{H} 2 / \mathrm{C} 2 \mathrm{H} 4 \mathrm{CH} 4 / \mathrm{H} 2$ and to the concentration of specific gases such as hydrogen, carbon monoxide, methane, ethane, ethylene, acetylene. Different combinations of these four codes represent different fault patterns, overheating, arcing, and corona. Fuzzy Analysis an integrated analysis, it can diagnose the fault of transformer effectively and manage the data of DGA in oil of transformer [19].

\section{NeURo-FuZZY ApPliCATION to DGA}

In new development in a transformer diagnostic system that utilized both an expert system and a neural network to detect different causes of failures in a power transformer [20] The knowledge of the expert system has many uncertainties $\&$ problems, and therefore fuzzy logic is employed. In this case, the neural network employs sampled learning to complement the knowledge-based diagnosis of the expert system. The two techniques are integrated by comparing the expert system conclusion with the neural network reasoning using a consultative mechanism. A block diagram for this type of hybrid system is given in Fig. 3.

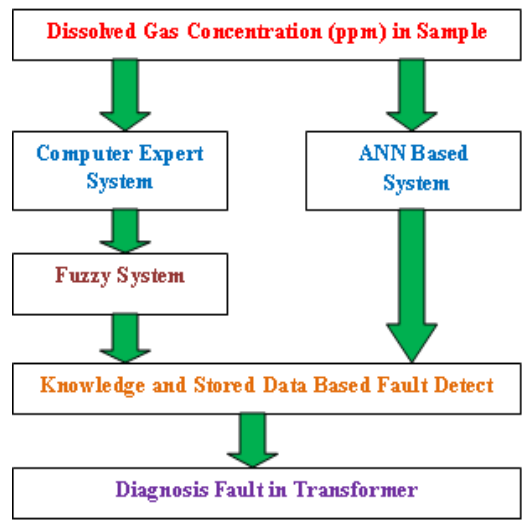

Fig.3. Strategy for combined fuzzy logic, expert system, and neural network

The advantage of neuro - fuzzy modelling includes the facility for extracting the information or knowledge hidden in the measured data and the ability for approximating complicated non linear functions with simpler models [21].

\section{EXTENSION NeURAL NETWORK APPLICATION TO DGA}

There are many methods to detect the major fault by the help of dissolved gas in transformer oil. The ENN proposed is a Combination of extension theory and a neural network. Using an innovative extension distance instead of Euclidean distance (ED) to measure the similarity between tested data and the cluster centre, it can effect supervised learning and achieve shorter learning times than traditional neural networks. To overcome the drawbacks of traditional neural networks, a type of neural network is proposed for incipient fault diagnosis of power transformers in this paper. The proposed ENN uses a combination of extension theory (IS) and the neural network. In this way there are some classified problems that feature the defined range [22].

\section{SELF ORGANIZING MAP APPLICATION TO DGA}

The SOM approach has provided a means of enhancing the condition monitoring of power transformers. It is more useful than other techniques for the diagnosis of transformer faults. This method is based on the data mining methodology and the self-organizing map, has been compared and validated using conventional interpretation schemes and real fault cases, thereby proven to be capable of enhancing the condition monitoring of power transformers. Basically the approach based on the self organizing map algorithm and Self- Organizing Polynomial network techniques uses the real fault cases for the present position of power transformer [23]. That SOM approach requires only recorded DGA data for modelling and it is able to provide convincing diagnosis to all DGA records for the easy and cost effective to implement and eliminate the elements of uncertainty and ambiguity. Basically that effective approach can be used as a decision support tool by expert engineers, in addition to conventional approaches and expert experiences, to monitor and diagnose the operating condition of power transformers.

The self-organizing polynomial networks based transformer diagnosis system has been implemented based on the actual diagnostic gas records collected in power transformer. SOPNs based diagnostic scheme to handle the numerical, complicated, and uncertain relationships of dissolved gases (input) to fault conditions (output). The developed diagnosis system can accurately capture the complex and subtle input-output relationships [24].

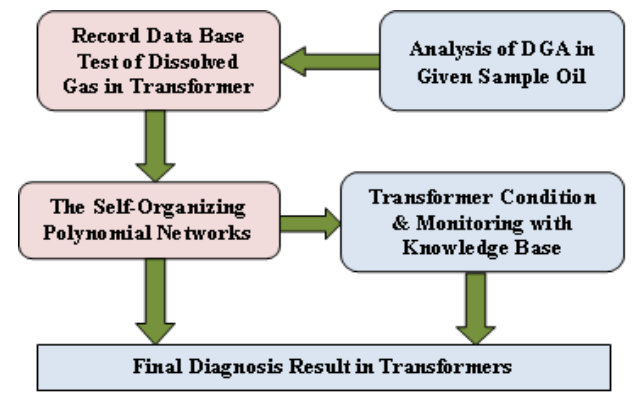

Fig. 4. The Self-Organizing Transformer Diagnosis System

The advantages of Self organizing map over traditional \& effective methods for analysis and interpretation of dissolved gas analysis data such as, that is not depend on any actual fault cases and DGA schemes for its modelling so it is very easy and cost effective to implement. That is not only allows diagnosis of a fault based on visualization of inherent data characteristics, but it also allows the Condition monitoring of power transformer [25].

\section{GeNETIC ALGORITHM APPLICATION IN DGA}

We can also prediction of dissolved gas in transformer oil concentration with using Genetic Algorithm (GA) and Grey theory [26]. Genetic algorithm can impose a series of genetic operations such as selection, crossover and mutation on the current population, which will generates the new population and gradually evolves to the optimal solution. Genetic Algorithm is defined as proceeds to initialize a population of solutions randomly, and then improves itself through iterations of selection, crossover and mutation. Genetic algorithm is a search technique used in computing to find exact or approximate solutions to optimization and search problems. 
In short, it is a very efficient and widely used technique to determine dissolved gases due to deterioration of the insulating oil of transformers.GA has been applied to search the optimal parameter of new technique grey model. In gray system some part of informations are known and some informations are unknown [27]. The main advantage of this system utilize only a few know data points by such a way of accumulated Generating Operation (AGO) to established the more effective model for diagnosis to power transformer. In such method has the main advantage, not to pursue large sample size and not require special data distribution. In regression analysis, calculation is very easy \& smaller than other methods.

The GA determined the dilation and translation parameters and the weighting values of the most accurate diagnosis DGA model. The information hidden in the many DGA diagnostic records was efficiently extracted by the GA without heavy human involvement, because of the self-tuning, multiscale, multiresolution, and localization of the method [28].

\section{WAVELET NeTWORKS APPLICATION TO DGA}

The wavelet network has attracted much attention in signal analysis and pattern recognition. The wavelet theory has found many effective applications in function approximation, numerical analysis, and signal processing. Some time we can combine the wavelet multi scale theory and neural network and get a novel high performance network-wavelet networks, which are easy-to identify network structures and deal effectively with the problems of high dimensional model. Some studies have reported the use of wavelet network and DGA samples for incipient fault detection in power transformers. The $\mathrm{WN}$, trained by an error back propagation algorithm or binary encoding genetic algorithm (GA) had good diagnosis capabilities.

WN approaches famous those of the conventional back-propagation neural-network method and are suitable for faults diagnosis of power transformers, especially with the evolving WN achieving superior performance. We can divide the feed forward wavelet network commonly used into two types, then these two types of wavelet networks based on two different activation functions of the wavelet nodes are applied in fault diagnosis of power transformers. Test results verified that the WNs possess higher diagnosis accuracy and require less learning time than the existing methods [29-30].

\section{RECENT TECHNIQUES FOR DGA}

Many researchers are working for development of new techniques for this important analysis. Some of the techniques are hybrid of the above mentioned procedures. The research work is still in progress to develop DGA techniques based upon new principals.

Jiyin Zhao et al. has explained transformer Fault Diagnosis Based on Niche Genetic Algorithm by using optimize Adaptive-Learning-Rate-Momentum Back Propagation (BP) Network [31]. The reasoning process of Bayesian Network (BN) is fast calculating process of probability based on already existent information. This technique find out power transformer faults rapidly and exactly into transformer fault diagnosis by constructing the transformer fault diagnosis model [32, 33].

A fault system of power transformer can be also recognized as a typical grey system. In this new method grey target theory is given to recognized power transformer state without the any standard fault model. Grey system theory is used to study the uncertain system having the characteristics of small sample and uncertainty poor information. It uses partial given information to realize the object and get its evolution rule. It provides an available method for state assessment of power transformer. It is one of the effective methods for Multi-objective decision-making. [34].

A fault diagnosis model of DGA based on an improved binary tree multi -class support vector machine is constructed. This method rapidness and precision than other methods and better improvement in accuracy rate of fault diagnosis. In this operational models of the oil-immersed transformer are divided into five models: thermal fault of low temperature, thermal fault of high temperature, discharge of low energy, discharge of high energy and normal state. These faults are consisted of six types of dissolved gases (H2, CH4, C2H6, $\mathrm{C} 2 \mathrm{H} 4, \mathrm{C} 2 \mathrm{H} 2$ and $\mathrm{CO}$ ) in transformer oil [35].

Laser Raman Spectroscopic Analyses approach used for Dissolved Gases in power transformer oil [36]. A laser excites a target, and the spectrum of the energy-shifted, back-scattered radiation serves as a "fingerprint" - providing compositional and structural information.

A novel extension method for power equipment fault diagnosis is presented based on a least square (LS) fusion combining neural network and dissolved gas analysis (DGA) [37]. In that method Compared with single neural network, the LS weighted fusion combining neural network can identify equipment fault correctly when the DGA characteristics is very similar with other type fault, while the single neural network may fail at this case. The identification correct ratio of the combining network is much higher than single neural network.

An innovative method is presented for fault diagnosis of power transformers, which is based on the matter- element model and Extended Relation Function (ERF) [38]. In this test gas results show that this method can not only diagnosis the main fault types of transformers, but it can also detect important information future trends and multi-fault analysis by the relation degree [39].

In modern trends Dissolved Gas Analysis in transformer by the help of new diffusion dynamic model. The method is based on the measurement of diffusion times of characteristic gases inside the oil-impregnated cellulose. In that method has been used to obtain the model parameters from limited experimental data [40]. Characteristic gases dissolved in transformer oil migrate between the liquid and solid insulation. Fick's law of diffusion dictates this process in the most fundamental way. The dynamic model used in this study was first suggested by von Guggenberg and Melcher for moisture diffusion [41]. Application of this model to characteristic gases, however, requires experimental acquisition of the model parameters for these gases.

A Fault Diagnosis Method Combined Fuzzy Logic with CMAC (Cerebellar model articulation controller) Neural Network for Power Transformers. It uses fuzzy logic to extract diagnosis rules from a lot of fault samples, and then, the extracted rules are employed to optimize CMAC network. Many real fault samples are analyzed by FCMAC (Fuzzy 
CMAC neural network) for the purpose of verification, and the analyzed results are also compared with those analyzed by IEC ratio method and those by the CMAC neural network [42].

A new technique developed on-line monitoring system to detect the concentrations of $\mathrm{H} 2$ and $\mathrm{CO}$ dissolved in transformer oil. In this system mounts Polyperfluoro ethylene-propylene membrane, electrochemical gas sensors, a wireless communication terminal based on RF (Radio Frequency) transceiver, and data management software recording the concentration of $\mathrm{H} 2$ and $\mathrm{CO}$. Those systems which incorporated wireless data acquisition will taken definitely shorten a time gap between the changes of gas concentrations and dissolved gas analysis. In facts, the whole system is able to rapidly determine concentration of dissolved $\mathrm{H} 2$ and $\mathrm{CO}$, and definitely provides the results instantaneously by using wireless data acquisition [43].

A novel association rule mining (ARM)-based dissolved gas analysis (DGA) approach to fault diagnosis of power transformers. In typical knowledge discovery process includes three steps, i.e. preprocessing data, applying a data mining algorithm, and post processing obtained results. In facts, such a ARM process are developed, including data preprocessing, rule discovery Apriori-Total From Partial TFP, and association rule set ARS post processing. The final results demonstrate that the novel ARM-based DGA approach has achieved the highest fault diagnosis accuracies [44].

The DGA real-time monitoring system developer needs to reduce the sampling interval of a real-time DGA monitoring system. So a new methodology developed such as, constructed upon Dempster - Shafer theoretic approach. That is proposes a novel interpretation rule and algorithm. Weibull functions were utilized to quantify the degree of belief in major fault according to respective DGA parameters. Threshold belief mass (THB) that distinguishes incipient fault from major fault, which is located within the interval of degree of belief in major fault were observed. In this approach, based on minimum threshold values, a novel rule termed as Threshold Belief Mass (THB) interpretation rule is proposed and compared with Dempster's and Yager's combination rule. In fact, this new approach enables a system operator to interpret DGA data more systematically [45].

New Quantum Neural Network (QNN) was applied to diagnosis the transformer fault by employing the DGA data. The advantage of the QNN model based on quantum phase in dealing with uncertain, ambiguous and cross fault data are verified by the practical engineering application [46]. Genetic

Programming (GPFM) is proposed to diagnose the fault types of insulation of power transformers. GPFM algorithms, an insulation fault diagnosis system for power systems is designed to distinguish the insulation fault types of power transformers [47].

\section{CONCLUSION}

DGA is a very efficient tool for diagnosing incipient faults in oil-filled electrical equipment. This paper has presented the application of various AI techniques like expert system ES, ANN, GA, Fuzzy system and WNs approach etc for dissolved gas analysis. The paper has critically examined the basic principle procedure and application for DGA of Power transformer. It may conclude that these methods are much better than the classical methods in particularly for interpretation of the DGA results.

\section{ACKNOWLEDGMENT}

The authors are thankful to Technology Information Forecasting and Assessment Council and Centers of Relevance \& Excellence (TIFAC-CORE) on Power Transformer Diagnostics and Dr. R. K. Jarial, Associate Professor and Office-in-charge, HV Lab, NIT Hamirpur for providing necessary infrastructural facilities for carrying out the research work.

\section{REFERENCES}

[1] IS 10593:2006, Indian Standard IEC60599: "Oil-filled electrical equipment Sampling of gases and of oil for analysis of free and dissolved gases - Guidance", Third edition, 2005-06.

[2] ANSI/IEEE Standard C57.104-1991: "IEEE guide for the interpretation of gases generated in Oil-Immersed Transformers".

[3] CEI, IEC 60599: "Mineral Oil Impregnated Electrical Equipment in service. Guide to the interpretation of Dissolved Free Gas Analysis", second edition 1999-03.

[4] M. Duval, "A review of fault detectable by gas-in-oil analysis in transformer", IEEE electrical Insulation magazine 2002, 10(3), pp. 8-17, 2002.

[5] K. F. Thang, R. K. Aggarwal, A. J. MacGrail, and D. G. Esp, "Application of Self-Organizing Map Algorithms for Analysis and Interpretation of Dissolved Gases in Power Transformers", IEEE Power Engineering Society Summer Meeting, Vancouver, BC, Canada, 3, pp. 1881-1886, 2001.

[6] K. F. Thang, R. K. Aggarwal, D. G. Esp and A. J. MacGrail, "Statistical and Neural Analysis of Dissolved Gases in Power transformers", Eighth International Conferences on Dielectric Materials, Measurements and Application (IEE Conf. Publ. No. 473), Edinburgh, UK 2007, pp. 1-9, 2007.

[7] CIGRE WG A2.18: "Guide for Life Management Techniques for Power Transformers", 20th January, 2003.

[8] Diego Roberto Moaris, and Jacqueline Gisete Rolim, "An artificial Neural Network Approach to Transformer Fault diagnosis", IEEE Transactions on Power Delivery, 11(4), pp. 1836-1841, 1996.

[9] IS 10593:2006 Indian Standard: "Mineral Oil-impregnated Electrical Equipment in service-Guide to interpretation of dissolved and free gas analysis".

[10] Amritpal Singh, and P. Verma, "A Review of Intelligent Diagnostic Methods for Condition Assessment of Insulation System in Power Transformers", IEEE, International Conference on Condition monitoring and Diagnosis, Beijing, China, 2008.

[11] N. K. Patel and R .K. Khubchandani, "ANN based power transformer fault diagnosis", IE(I) Journal-EL 2004.

[12] S Das, and O. Olurotimi Noise, "Recurrent Neural Networks: The Discrete-time case", IEEE Transactions on Neural Networks 1998, 9(5), pp. 937-946, 1998.

[13] W. Xu, D. Wang, Z. Zhou and H. Chen, "Fault diagnosis of power transformers: application of fuzzy set theory, expert systems and artificial neural networks”, IEE Proc.-Sei. Meas. Technol 1997, 144(1), 1997.

[14] Y. Zhang, X, Ding, Y. Liu, and P.J. Griffin, “An Artificial Neural Network Approach to Transformer Fault Diagnosis", IEEE Transactions on Power Delivery, 11(4), pp. 1836, 1996.

[15] N. Yadaiah, L. Sivakumar., B. L. Deekshatulu and V. Sri Hari Rao, "Neural Network Architectures for Describing Nonlinear Input-Output Relations", Electronic Modeling, 24(3), pp. 48-61, 2002.

[16] N. Yadaiah and Ravi Nagireddy, "Fault Detection Techniques for Power transformers", ICPS 2007, IEEE/IAS 6, pp. 1-9, 2007.

[17] A. Singh and P. Verma, "A review of intelligent diagnostic methods for condition assessment of insulation system in power transformers" Condition Monitoring and Diagnosis, 2008. CMD 2008. International Conference, pp. 1354 - 1357, 2008.

[18] Yann-Chang. Huang, Tzer Ym Hong and Ching-Lien Huang, "Developing a New Transformer Fault Diagnosis System through Evolutionary Fuzzy Logic", IEEE Transactions, 12(2), pp 761-767, 1997. 
[19] Teo. Brescia, Sergio. Bruno, La Scala Massimo, Silvia Lamonaca, Giuseppe Rotondo, and Ugo Stecchi, "A fuzzy-logic approach to preventive maintenance of critical power transformers", Electricity Distribution, 20th International Conference and Exhibition , pp. 1 - 5, 2009.

[20] W. Xu, D. Wang, Z. Zhouand and H. Chen, "Fault Diagnosis of Power Transformers: Application of Fuzzy Set Theory, Expert Systems, and Artificial Neural Networks", IEEE Proceedings of Science, Measurement and Technology, 144(1), pp. 39-44, 1997.

[21] H. Ding, "An expert system for large power transformer fault diagnosis, Expert System application to Power Systems" IV Proceedings1992, UK, pp- 499-502, 1992.

[22] M.-H. Wang, "Extension neural network for power transformer incipient fault diagnosis, Generation, Transmission and Distribution", IEE Proceedings 2003, 150(6), pp. 679-685, 2003.

[23] Hong-Tzer. Yang, and Yann-Chang. Huang, "Intelligent Decision Support for Diagnosis of Incipient Transformer Faults Using Self-organizing Polynomial Networks", Power Industry Computer Applications, 20th International Conference 1997, pp 60 - 66, 1997.

[24] K. F. Thang, R. K. Aggarwal, A. J. McGrail, and D. G. Esp, "Analysis of Power Transformer Dissolved Gas Data Using the Self-Organizing Map”, IEEE transactions, 18 (4), pp. 142-149, 2003.

[25] Jashan. Deep Singh, Yog Raj Sood, and Piush Verma, "Review of Various Dissolved Gas Analysis (DGA) Techniques for Power Transformer", The Icfai University Journal of Electrical \& Electronic Engineering 2008, 1(4), pp. 60-69, 2008.

[26] Wang Yongqiang, and Lu. Fangcheng, "Fault Diagnosis Method of Capcitive Equipment Based on Grey Relational Analysis", 9th International Conference on Properties and Applications of Dielectric Materials, Harbin, China, 2009.

[27] Bin Song, Ping Yu, Yunbai Luo and Xishan Wen, "Study on the Fault Diagnosis of Transformer Based on the grey Relational Analysis", IEEE International Conference on Power system Technology, 4, pp. 2231-2234, 2002

[28] K. F. Thang, R. K. Aggarwal, A. J. McGrail and D. G. Esp, "Analysis of Power Transformer Dissolved Gas Data Using the Self-Organizing Map”, IEEE Transactions on Power Delivery, 18(4), 2003.

[29] Yann-Chang Huang, "A New Data Mining Approach to Dissolved Gas Analysis of Oil-Insulated Power Apparatus", IEEE Transactions on Power Delivery, 18(4), pp.1257-1261, 2003.

[30] Chen Weigen, Pan Chong, Yun Yuxin, and Liu Yilu, "Wavelet Networks in Power Transformers Diagnosis Using Dissolved Gas Analysis", IEEE Transactions On Power Delivery 2009, 24(1), pp.187-194, 2009.

[31] Jiyin Zhao, Ruirui Zheng, and Haihong Dong, "Study on Power Transformer Fault Diagnosis Based on Niche Genetic Algorithm", IEEE, Natural Computation, Fifth International Conference, pp 436-440, 2009

[32] Wang Yongqiang, Lu Fangcheng, Li. Heming, and Li. Yanqing, "The fault diagnosis method for power transformer based on BN And DGA", Electrical Insulating Materials, Proceedings of 2005 International Symposium, 2, pp. $476-479,2005$.

[33] J.G. Rolim, P.C. Maiola, H.R, Baggenstoss, A.R.G: da Paulo, "Bayesian Networks Application to Power Transformer Diagnosis", Power Tech, IEEE Lausanne 2007, pp. 999-1004, 2007.

[34] Li. Jianpo, Chen Xiaojuan, and Wu. Chunming, "Power Transformer State Assessment Based on Grey Target Theory", Measuring Technology and Mechatronics Automation, 2009. ICMTMA '09, International Conference, pp. $664-667,2009$.

[35] Sun Xiaoyun, An. Guoqing, Fu. Ping, and Bian. Jianpeng, "Fault diagnosis model of power transformer based on an improved binary tree and the choice of the optimum parameter of multi-class SVM", Intelligent Computing and Intelligent Systems, IEEE International Conference, pp. $567-571,2009$.

[36] S. N. White, "Laser Raman Spectroscopic Analyses of Dissolved Gases", IEEE Conference, Oceans, pp.1 - 6, 2007.

[37] Lv. Ganyun and Wang. Xiaodong, "Fault Diagnosis of Power Equipment Based On Dissolved Gas Analysis And LS Fusion Combining Neural Network", Natural Computation, ICNC 2007, IEEE, Third International Conference, 1, pp.154-158, 2007.

[38] Wang Mang-Hui. "A Novel Extension Method for Transformer Fault Diagnosis", IEEE Transactions on Power Delivery, pp. 164 169, 2003

[39] Limin Zhang., Li. Zheng, Ma. Hongzhong, and P. Ju, "Power transformer fault diagnosis based on extension theory", Electrical Machines and Systems, ICEMS, IEEE, Proceedings of the Eighth International Conference, pp. 1763-1766, 2005.
[40] A. Shahsiah, R.C. Degeneff and J.K. Nelson, "A New Dynamic Model for Propagation of Characteristic Gases in Transformer Oil-Cellulose Structures Due to Temperature Variations", Annual Report Conference on electrical Insulation and dielectric Phenomena, pp. 444-447, 2005 .

[41] P. A. von. Guggenberg, and J. R. Melcher, "Moisture dynamics in paper/oil systems subject to thermal transients", EPRI, report No. EL-6918, 1990.

[42] Zhao Xiaoxiao and Yun Yuxin, "A Fault Diagnosis Method Combined Fuzzy Logic with CMAC Neural Network for Power Transformers", Pattern Recognition, 2009 (CCPR 2009), Chinese Conference, pp. 1 $5,2009$.

[43] Zhao Xuezeng and Li. Yangliu "An On-Line Monitoring System for Gases Dissolved in Transformer Oil Using Wireless Data Acquisition", IEEE conference, Power and Energy Engineering Conference, APPEEC 2009. Asia-Pacific, pp. 1 - 4, 2009.

[44] Z. Yang, W.H. Tang, A. Shintemirov and Q.H. Wu, "Association Rule Mining-Based Dissolved Gas Analysis for Fault Diagnosis of Power Transformers", Systems, Man, and Cybernetics, Part C: Applications and Reviews, IEEE Transactions 2009, 39(6), pp. 597-610, 2009.

[45] A. Mohd Radzian and M. Itoh, "Decision making framework for power transformer Dissolved Gas Analysis on the basis of Dempster-Shafer theoretic approach", IEEE Conference of Prognostics and Health Management, pp. 1-7, 2010.

[46] Cai Guowei Liu Ning and Yang Deyou, "The transformer fault diagnosis based on Quantum Neural Network", International Conferences on Computer, Mechatronics, Control and Electronic Engineering (CMCE), IEEE, 2010 (4), pp. 396 - 400.

[47] Zheng Zhang, Kangling Fang and Weihua Huang, "A Genetic Programming Based Fuzzy Model for Fault Diagnosis of Power Transformers", 3rd International Conference on Intelligent Networks and Intelligent Systems (ICINIS), IEEE, 2010, pp. $455-458$.

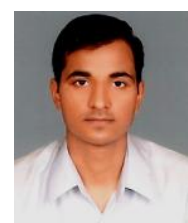

Naveen Kumar Sharma, (Member IACSIT \& Student Member IEEE) was born in Kanpur (Uttar Pradesh), India. $\mathrm{He}$ obtained his B.Tech in Electrical \& Electronics Engineering in 2008 from Utter Pradesh Technical University Lucknow (U.P.). He received his M. Tech degree in Power System from National Institute of Technology Hamirpur (H.P.)-India, in 2010. Presently he is pursuing Ph.D from Department of Electrical Engineering, National Institute of Technology Hamirpur(HP).His research interests are in the area of Power System, power transformer diagnosis, power sector restructuring and deregulation, renewable energy.

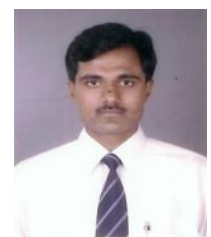

Prashant Kumar Tiwari, (Member IACSIT \& Student Member IEEE) obtained his B.Tech degree in Electrical Engineering with "Honours" from U.P.T.U. Lucknow (india) in 2007. He received his M. Tech degree from National Institute of Technology Hamirpur(HP)-India, in 2009. Presently he is pursuing Ph.D from Department of Electrical Engineering, National Institute of Technology Hamirpur(HP). His research interests are in the area of Powe System, FACTS, power sector deregulation.

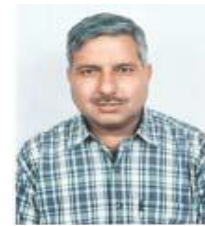

Yog Raj Sood,(Senior Member IEEE \& Member IACSIT) received his B.E. degree in Electrical Engineering with "Honours" and M.E. in Power System from Punjab Engineering College Chandigarh (U.T.), in 1984 and 1987 respectively. He has obtained his Ph.D. from Indian Institute of Technology (IIT), Roorkee in 2003. He joined Regional Engineering College Kurukshetra in 1986. Presently he is working as Professor in the Electrical Engineering Department of National Institute of Technology, Hamirpur (H.P.) India since January 2006. He has been honoured with many awards, prizes, appreciation letters/certificates for his excellent research, academic and administrative duties. He has published a number of research papers. His research interests are in the area of power sector deregulation, renewable energy, condition monitoring of power transformer etc. 